\title{
Patient-Controlled Analgesia for Cancer-Related Pain: Clinical Predictors of Patient Outcomes
}

\author{
Emily J. Martin, MDa; Eric J. Roeland, MDa; Madison B. Sharpª Carolyn Revta, MPH ${ }^{\mathrm{a}}$; \\ James D. Murphy, MD ${ }^{\mathrm{b}}$; Katherine E. Fero ${ }^{\mathrm{b}}$; and Heidi N. Yeung, MD
}

\begin{abstract}
Background: Patient-controlled analgesia (PCA) is an effective approach to treat pain. However, data regarding patterns of PCA use for cancer pain are limited. The purpose of this study was to define the patterns of PCA use and related outcomes in hospitalized patients with cancer. Methods: We identified 90 patients with cancer admitted to a single academic center who received PCA for nonsurgical, cancerrelated pain and survived to discharge between January 2013 and January 2014. Data collected included patient demographics, cancer diagnosis, type of cancer-related pain, PCA use, opioid-specific adverse events, and 30-day readmission rates for pain. Univariable and multivariable linear regression models were used to analyze the association between patient and clinical variables with PCA duration. Logistic regression models were used to evaluate the relationship between patient and clinical variables and 30-day readmission rates. Results: The median length of hospitalization was 10.2 days with a median PCA duration of 4.4 days. Hematologic malignancies were associated with longer PCA use $(P=.0001)$, as was younger age $(P=.032)$. A trend was seen toward decreased 30-day readmission rates with longer $P C A$ use $(P=.054)$. No correlation was found between 30 -day readmission and any covariate studied, including age, sex, cancer type (solid vs hematologic), pain type, palliative care consult, or time from PCA discontinuation to discharge. Conclusions: This study suggests that there is longer PCA use in younger patients and those with hematologic malignancies admitted with cancer-related pain, with a trend toward decreased 30-day readmission rates in those with longer PCA use.
\end{abstract}

J Natl Compr Canc Netw 2017;15(5):595-600

\section{Background}

Pain is prevalent among patients with cancer, impacting $30 \%$ to $60 \%$ of patients at initial diagnosis, $>30 \%$ of patients after curative treatment, and $70 \%$ to $80 \%$ of patients with advanced disease. ${ }^{1-3}$ Approximately threefourths of cancer-related pain is attributed to the direct effects of the disease, with the remaining one-fourth attributed to side effects of therapy. ${ }^{4}$

Opioids are the mainstay of treatment for both acute and chronic cancer-related pain. For the past 30 years, the WHO analgesic ladder has served as a validated framework for the use of opioids in the management of cancer pain. ${ }^{5}$ Despite the proven effectiveness

From the aivision of Palliative Medicine and Hematology/Oncology, Department of Internal Medicine, and 'Department of Radiation Medicine and Applied Sciences, UC San Diego Moores Cancer Center, La Jolla, California.

Submitted August 17, 2016; accepted for publication January 3, 2017.

The authors have disclosed that they have no financial interests, arrangements, affiliations, or commercial interests with the manufacturers of any products discussed in this article or their competitors.

Author contributions: Study conception: Martin, Roeland, Yeung. of opioids, 1 of 2 patients with cancer continues to experience undertreated pain, and approximately $25 \%$ of patients with cancer experience severe pain at the time of death. ${ }^{6,7}$ Insufficient use of opioids both within the United States and worldwide has been shown to be the primary reason cancer pain remains undertreated in both outpatient and inpatient settings..$^{8-11}$

Pain is a leading cause of emergency department visits and unscheduled hospital admission within the cancer population. ${ }^{12,13}$ Moderate to severe pain scores among patients with cancer in the ambulatory setting have been shown to be predictive of hospitalization within 30 days and are also associated with prolonged duration of admission. ${ }^{14}$ Nationwide, $>60 \%$ of patients

Methodology: Martin, Roeland, Revta, Yeung. Investigation: Martin, Sharp, Yeung. Formal analysis: Murphy, Fero. Drafting of manuscript: Martin, Yeung. Review and editing: Martin, Roeland, Yeung. Project administration: Revta.

This manuscript was presented at The Annual Assembly: Hospice and Palliative Care; March 9-12, 2016; Chicago, Illinois.

Correspondence: Heidi N. Yeung, MD, UC San Diego Moores Cancer Center, Division of Palliative Medicine and Hematology/Oncology, 3855 Health Sciences Drive, MC 0987, La Jolla, CA 92093. E-mail: hnyeung@ucsd.edu 
with end-stage cancer are admitted to the hospital within the last month of life and $>40 \%$ are admitted to the intensive cancer unit within the last 3 months of life..$^{15}$ Despite the prevalence of clinically significant pain among hospitalized patients with cancer, studies consistently show that opioids are underutilized in the inpatient setting. ${ }^{16,17}$

Patient-controlled analgesia (PCA) is a pain medication delivery system that enables a patient to safely self-administer a set dose of opioids on a predetermined schedule while maintaining the lowest serum concentration of the medication needed to achieve pain relief. Through frequent, small, incremental opioid doses, analgesia is achieved while risks of overdose, opioid toxicity, or gaps in analgesic coverage are minimized..$^{18} \mathrm{PCA}$ is associated with greater patient satisfaction, improved pain control, less overall opioid consumption, and a similar side effect profile compared with nurse-administered regimens or continuous opioid infusions. ${ }^{19,20}$

PCA is most commonly used in inpatient surgical services for management of postoperative pain, although it has been shown to be safe and effective in managing pain associated with angina, sickle cell crises, burn injuries, and, notably, cancer. ${ }^{11,19,21-25}$ Although early studies of hospitalized patients with cancer demonstrated $>95 \%$ pain control achieved with PCA, ${ }^{26,27}$ to our knowledge no published study has examined the clinical patterns of PCA use in adult inpatients with cancer-related pain and its effect on 30-day readmission rate or hospital length of stay. In this single-center, retrospective study, we evaluated PCA use and associated clinical outcomes in patients admitted to the hospital with cancerrelated pain, defined as acute or chronic physical pain attributed to cancer or its treatment.

\section{Methods}

\section{Study Cohort}

All patients with cancer who were admitted to University of California, San Diego (UCSD) Medical Center between January 2013 and January 2014 who received PCA during their hospitalization and survived to discharge were identified retrospectively using a single electronic medical record $(n=499)$. For patients admitted for $>1$ hospitalization, only their first hospitalization within the study period was analyzed $(n=272)$. Patients were excluded who received
PCA for non-cancer-associated pain $(n=16$; eg, pain associated with traumatic injury). Patients were also excluded if they received PCA for postsurgical pain $(n=166$; specifically, any patient who underwent a major surgical procedure during their hospitalization was excluded; we did not exclude patients who underwent a minor surgical intervention, including peripherally inserted central catheter line insertion, gastric tube placement, lumbar puncture, bronchoscopy, bone marrow biopsy, endoscopy, cystoscopy, or drain placement by Interventional Radiology). The remaining 90 patients constituted our primary analytic sample population.

\section{Outcomes Analyzed}

All medical records were reviewed and the following data were collected: age, sex, cancer diagnosis, type of cancer-associated pain (eg, abdominal pain, musculoskeletal pain, mucositis, other), duration of hospitalization, duration of PCA use, time from admission to PCA initiation, time from discontinuation of PCA to discharge, use of a basal infusion, opioid type, number of total opioid rotations, readmission for pain within 30 days of discharge, consultations by the palliative care service, regular use (defined as an average of $\geq 2$ doses daily) of intravenous or oral opioids while on PCA, and opioid-associated adverse events. Opioid-related adverse events were defined as respiratory depression requiring naloxone or mechanical ventilation; oversedation as measured by Richmond Agitation-Sedation Scale (RASS) scores of -3 to -4 or administration of naloxone; and discontinuation of PCA or change in PCA opioid type due to intolerable side effects (eg, nausea, vomiting, pruritus). To assess for any PCA-associated deaths, we performed a secondary analysis of all patients with cancer who were admitted to UCSD Medical Center between January 2013 and January 2014 who received PCA during their hospitalization but did not survive to discharge.

\section{Statistical Analysis}

Univariable and multivariable linear regression models were used to look for an association between patient and clinical variables with PCA duration. Logistic regression models were used to evaluate the relationship between patient and clinical variables and 30-day readmission rates. Differences between the patients in our primary cohort and those who 
died during hospitalization were assessed with $t$-tests for continuous variables (age) and Fisher exact test for categorical variables.

\section{Results}

Patient characteristics including age, sex, cancer site, and primary type of pain are outlined in Table 1. There were no statistically significant differences in these variables between the primary patient population and the cohort of deceased patients (age, $P=.44$; sex, $P=.83)$, cancer site $(P=.39)$, and primary type of pain $(P=.37)$. The inclusion of various cancer types is outlined in Table 1, with hematologic malignancies (including acute myeloid leukemia, acute lymphocytic leukemia, multiple myeloma, diffuse large B-cell lymphoma, chronic myeloid leukemia, Hodgkin lymphoma, non-Hodgkin's lymphoma, follicular

\begin{tabular}{|lc|}
\hline Table 1. Patient Characteristics & $\mathbf{n}(\%)$ \\
\hline Characteristic & 90 \\
\hline Number of patients & $55(43-63)$ \\
\hline Sexian age (IQR), y & $41(44.6 \%)$ \\
\hline Female & $49(54.4 \%)$ \\
\hline Male & \\
\hline Cancer type & $36(40.0 \%)$ \\
\hline Hematologic & $54(60.0 \%)$ \\
\hline Solid tumor & $10(11.1 \%)$ \\
\hline Gastrointestinal (colorectal) & $9(10.0 \%)$ \\
\hline Gastrointestinal (noncolorectal) & $6(6.7 \%)$ \\
\hline Gynecologic & $7(7.8 \%)$ \\
\hline Lung & $6(6.7 \%)$ \\
\hline Genitourinary & $6(6.7 \%)$ \\
\hline Head/Neck & $3(3.3 \%)$ \\
\hline Sarcoma & $2(2.2 \%)$ \\
\hline Breast & $2(2.2 \%)$ \\
\hline Neurologic & 0 \\
\hline Melanoma & $3(3.3 \%)$ \\
\hline Unknown & $36(40.0 \%)$ \\
\hline Musculoskeletal & $38(42.2 \%)$ \\
\hline Cutanest & $11(12.2 \%)$ \\
\hline
\end{tabular}

Abbreviation: IQR, interquartile range. lymphoma, myelodysplastic syndrome, polycythemia vera with myelofibrosis, and lymphoblastic lymphoma) constituting $40 \%(n=36)$ of the total patients on PCA. Most patients presented with either abdominal pain $(40 \%)$ or musculoskeletal pain $(42 \%)$.

Characteristics of the patient's hospitalizations and clinical courses, including duration of admission, presence or absence of palliative care consult, time between admission and start of PCA, duration of PCA use, and time between discontinuation of PCA and discharge/death, is detailed in Table 2. The median length of hospitalization was 10.2 days (interquartile range [IQR], 6.1-20.1), whereas the median duration on PCA was 4.4 days (IQR, 2.0-9.2).

Younger age was associated with longer duration of PCA use $(P=.032)$. A diagnosis of hematologic malignancy was also associated with longer duration on PCA $(P=.0001)$; this association between hematologic malignancies and duration of PCA use persisted despite exclusion of the patients with mucositis $(P=.001)$. There was also a trend toward decreased 30-day readmission rates with longer duration on PCA $(P=.054)$.

Description of the patterns of PCA use, including opioid type at the time of PCA initiation and discontinuation, number of patients who had a change to the PCA opioid type, use of basal infusion, and rate of adverse effects while on PCA, are detailed in Table 3. Hydromorphone was the initial opioid

\begin{tabular}{|c|c|}
\hline \multicolumn{2}{|c|}{ Length of hospitalization, days } \\
\hline Average (SD) & $15.1(12.5)$ \\
\hline Median (IQR) & $10.2(6.1-21.0)$ \\
\hline \multicolumn{2}{|c|}{ Length between admission and start of PCA, days } \\
\hline Average (SD) & $2.9(4.6)$ \\
\hline Median (IQR) & $0.6(0.2-2.8)$ \\
\hline \multicolumn{2}{|c|}{ Length of time on PCA, days } \\
\hline Average (SD) & $8.4(10)$ \\
\hline Median (IQR) & $4.4(2.0-9.2)$ \\
\hline \multicolumn{2}{|c|}{ Length of time between stop of PCA and discharge, days } \\
\hline Average (SD) & $3.8(5.3)$ \\
\hline Median (IQR) & $2.0(0.9-4.9)$ \\
\hline \multicolumn{2}{|c|}{ Palliative care consult } \\
\hline Yes & $37(41.1 \%)$ \\
\hline No & $53(58.9 \%)$ \\
\hline
\end{tabular}

Abbreviations: IQR, interquartile range; PCA, patient-controlled analgesia. 
Martin et al

\begin{tabular}{|c|c|}
\hline Characteristic & n (\%) \\
\hline \multicolumn{2}{|l|}{ Initial opioid used } \\
\hline Hydromorphone & $79(87.8 \%)$ \\
\hline Fentanyl & $6(6.7 \%)$ \\
\hline Morphine & $5(5.6 \%)$ \\
\hline \multicolumn{2}{|l|}{ Final opioid used } \\
\hline Hydromorphone & $75(83.3 \%)$ \\
\hline Fentanyl & $12(13.3 \%)$ \\
\hline Morphine & $3(2.2 \%)$ \\
\hline \multicolumn{2}{|l|}{ Change in PCA opioid type } \\
\hline Yes & $11(12.2 \%)$ \\
\hline No & $79(87.8 \%)$ \\
\hline \multicolumn{2}{|l|}{ Use of basal infusion via PCA } \\
\hline Yes & $47(52.2 \%)$ \\
\hline No & $43(48.8 \%)$ \\
\hline \multicolumn{2}{|c|}{ Basal infusion at time of PCA start } \\
\hline Yes & $29(32.2 \%)$ \\
\hline No & $61(67.8 \%)$ \\
\hline \multicolumn{2}{|c|}{ Basal infusion at time of PCA stop } \\
\hline Yes & $23(25.6 \%)$ \\
\hline No & $67(74.4 \%)$ \\
\hline \multicolumn{2}{|l|}{ Adverse events } \\
\hline Yes & $2(2.2 \%)$ \\
\hline Opioid-associated death & 0 \\
\hline Oversedation & $2(2.2 \%)$ \\
\hline Respiratory depression & 0 \\
\hline No & $88(97.8 \%)$ \\
\hline
\end{tabular}

Abbreviation: PCA, patient-controlled analgesia.

administered via PCA in $87.8 \%$ of patients $(n=79)$; 11 patients $(12.2 \%)$ had a change in the opioid type during their admission. Reasons for changing opioids included patient preference, lack of effect of prior opioid, or patient-reported side effects. Approximately half of patients $(52.2 \% ; n=47)$ received a basal infusion of opioid administered through PCA during their admission. One-third of patients $(32.2 \%$; $\mathrm{n}=29$ ) were started on basal infusion at the time of PCA initiation. Use of a basal infusion of opioid was associated with longer duration on PCA $(P<.0001)$ and longer hospital admission $(P=.0007)$; however, there was no correlation between use of a basal rate and 30-day readmission for pain $(P=.16)$. There was no relationship between coadministration of opioids (opioids received orally or intravenously in addition to those received via PCA) and PCA length
$(P=.14)$, admission length $(P=.11)$, or 30 -day readmission for pain $(P=.88)$. We also found no relationship between palliative care consult and duration on PCA $(P=.49)$, length of hospitalization $(P=.11)$, or 30 -day readmission for pain $(P=.12)$.

Two doses of naloxone were administered to a single patient due to oversedation while on PCA, but there were no other documented episodes of opioid toxicity or respiratory depression requiring respiratory support or naloxone. (The patient who received naloxone was a 73-year-old man with metastatic cholangiocarcinoma and renal failure who was admitted for persistent emesis and cancer-related pain. He was started on a hydromorphone PCA at admission, with no basal infusion ordered. He received a total of 3.6 mg of hydromorphone via PCA over the 12 hours before administration of naloxone. His oversedation in the setting of receiving opioids was suspected to be secondary to his impaired renal function.)

Of the 27 patients who received PCA during their hospitalization and died before discharge, all had been formally transitioned to comfort care status before their death; none of the patients died of opioidrelated complications. At the time of death, 14 of these patients had active PCA orders, whereas the remaining 13 patients had been transitioned to continuous opioid infusions because they were no longer able to self-administer opioids via PCA.

\section{Discussion}

This single-center, retrospective study examined the patterns of PCA use and associated outcomes in hospitalized patients with cancer-related pain. We found that younger age and a diagnosis of hematologic malignancy were associated with longer duration on PCA. We also identified a trend toward decreased 30-day readmission rates with longer duration on PCA.

Several studies have shown an association between younger age and severity of pain among patients with cancer. ${ }^{28,29}$ Our study demonstrated that younger age was associated with longer duration on PCA, suggesting that longer PCA use may be a function of multiple factors, such as more severe pain, cancer biology, opioid metabolism, tolerance, and/or ability to cope. This is supported by previous data showing that the length of time needed to achieve adequate pain control among inpatients with 
cancer increases with higher pain severity scores, and that younger patients in both the cancer and noncancer settings require higher doses of opioids to achieve analgesia. ${ }^{30,31}$ This finding, if reproducible in larger prospective studies, may have clinical implications regarding the approach to pain management in young adults with cancer.

Pain severity has also been shown to vary based on cancer type, with head and neck cancer being associated with the highest pain intensity scores. ${ }^{14} \mathrm{No}$ tably, a 2007 meta-analysis of the prevalence of pain among patients with cancer found no statistically significant association between pain prevalence and cancer type; however, this review assessed pain in patients with solid tumors and did not include those with hematologic malignancies. ${ }^{3}$ Our findings suggest, like younger age, that hematologic malignancies are associated with longer duration of PCA use. Again, using duration of PCA as a proxy for severity of pain among other factors, our findings are consistent with reports of persistent and challenging pain among patients with hematologic malignancies. ${ }^{32-34}$ Because it is well-established that mucositis is a painful and prolonged side effect of chemoradiation therapy in patients with hematologic disease that may also prevent oral administration of medications, we performed a subset analysis excluding patients who received PCA for pain secondary to mucositis. Interestingly, the association between hematologic malignancies and longer PCA duration persisted, despite exclusion of the patients with mucositis.

Given the intense pressures on healthcare organizations to improve the quality of care and clinical outcomes while maximizing efficiency and decreasing length of stays and hospital readmissions, many may be surprised by the finding that longer PCA use for cancer-related pain trended toward a decrease in 30-day hospital readmission for pain. We have observed that many hospitalists are concerned about initiating PCA for cancer pain management given concerns that it will prolong their hospitalization. Although we did not complete a formal analysis comparing patients with cancer receiving PCA versus those who did not, it is important to note that the average PCA use was slightly longer than a week and completed roughly 4 days before discharge (Table 2). We hypothesize that the ability to quickly titrate and determine opioid needs via PCA may serve as a more important approach to ensure the transition from intravenous to oral opioids is successful and may explain the observed trend in decreased 30-day readmission rate for pain. We aim to further explore PCA use, transitions to nonintravenous routes, and quality measures in future prospective trials.

Limitations of this study include its single-center, retrospective design and lack of formal comparison group. Although the visual analog scale is a wellrecognized and validated tool for pain assessment, given the variability in charting of pain scores in medical records, level of analgesia was not analyzed in this study (although discharge was assumed to be a marker of adequate pain control). There are also potential confounding variables not incorporated into this study, including the patient's disease stage, use of PCA during prior hospitalizations, preadmission opioid regimens (which were inconsistently documented and quantified in the medical record), and measurement and analysis of total oral morphine equivalents.

\section{Conclusions}

Our results suggest an association between longer duration of PCA use with younger age and hematologic malignancies. It is possible that this association may indicate unique characteristics of pain experienced by these patients and/or may indicate a more difficult transition to alternate regimens in these patients. To help clarify the role of PCAs in the management of cancer-related pain, further studies comparing the use of PCAs versus alternative opioid regimens (including oral and nurse-administered intravenous regimens) are needed. These studies should be aimed at determining specific recommendations regarding whether more aggressive oral opioid or nonopioid adjuvant medications added earlier in the course impacts length of stay or readmission for pain and whether early ( $<24$ hours from time of admission) palliative care consults affect these parameters.

\section{References}

1. Patrick D, Ferketich S, Frame $P$, et al. National Institutes of Health State-of-the-Science Conference Statement: symptom management in cancer: pain, depression, and fatigue, July 15-17, 2002. J Natl Cancer Inst 2003;95:1110-1117.

2. Esphani N, Bruera E. Current trends in cancer pain management. In: de Leon-Casasola OA. Cancer Pain: Pharmacological, Interventional, and 


\section{Martin et al}

Palliative Approaches. Philadelphia, PA: Saunders Elsevier; 2006;17.

3. Van den Beuken-van Everdingen M, De Rijke J, Kessels A, et al. Prevalence of pain in patients with cancer: a systematic review of the past 40 years. Ann Oncol 2007;18:1437-1449.

4. Meier DE, Isaacs SL, Hughes R, eds. Palliative Care: Transforming the Care of Serious Illness, Volume 33. Hoboken, NJ: John Wiley \& Sons; 2011.

5. Zech DF, Grond S, Lynch J, et al. Validation of World Health Organization Guidelines for cancer pain relief: a 10-year prospective study. Pain 1995;63:65-76.

6. Deandrea S, Montanari M, Moja L, Apolone G. Prevalence of undertreatment in cancer pain. A review of published literature. Ann Oncol 2008:1985-1991.

7. Foley KM. How well is cancer pain treated? Palliative Med 2011;25:398401.

8. Greco MT, Roberto A, Corli O, et al. Quality of cancer pain management: an update of a systematic review of undertreatment of patients with cancer. J Clin Oncol 2014;32:4149-4154.

9. Jacobsen R, Liubarskiene Z, Moldrup C, et al. Barriers to cancer pain management: a review of empirical research. Medicina (Kaunas) 2009;45:427-433.

10. McCaffery M. Pain control. Barriers to the use of available information. World Health Organization Expert Committee on Cancer Pain Relief and Active Supportive Care. Cancer 1992;70(5 Suppl):1438-1449.

11. Citron ML, Kalra J, Seltzer VL, et al. Patient-controlled analgesia for cancer pain: a long-term study of inpatient and outpatient use. Cancer Invest 1992;10:335-341.

12. Sadik M, Ozlem K, Huseyin M, et al. Attributes of cancer patients admitted to the emergency department in one year. World J Emerg Med 2014:5:85-90.

13. Vandyk AD, Harrison MB, Macartney G, et al. Emergency department visits for symptoms experienced by oncology patients: a systematic review. Support Care Cancer 2012;20:1589-1599.

14. Wagner-Johnston ND, Carson KA, Grossman SA. High outpatient pain intensity scores predict impending hospital admissions in patients with cancer. J Pain Symptom Manage 2010;39:180-185.

15. Bekelman JE, Halpern SD, Blankart CR, et al. Comparison of site of death, health care utilization, and hospital expenditures for patients dying with cancer in 7 developed countries. JAMA 2016;315:272-283.

16. McMillan SC, Tittle M, Hagan S, Laughlin J. Management of pain and pain-related symptoms in hospitalized veterans with cancer. Cancer Nurs 2000;23:327-336.

17. Greco MT, Roberto A, Corli O, et al. Quality of cancer pain management: an update of a systematic review of undertreatment of patients with cancer. J Clin Oncol 2014;32:4149-4154.

18. Lehmann KA. Recent developments in patient-controlled analgesia. J Pain Symptom Manage 2005;29:72-89.
19. McNicol ED, Ferguson MC, Hudcova J. Patient controlled opioid analgesia versus non-patient controlled opioid analgesia for postoperative pain. Cochrane Database Syst Rev 2015:CD003348.

20. Clarkson JE, Worthington HV, Furness $S$, et al. Interventions for treating oral mucositis for patients with cancer receiving treatment. Cochrane Database Syst Rev 2010:CD001973.

21. Mulligan MA. The innovative use of a patient-controlled analgesia pump for the management of cardiac angina. J Palliat Care 1998;14:47-49.

22. van Beers EJ, van Tuijn CF, Nieuwkerk PT, et al. Patiententrolled analgesia versus continuous infusion of morphine during vasooclusive crisis in sickle cell disease, a randomized controlled trial. Am J Hematol 2007;82:955960.

23. Ashburn MA. Burn pain: the management of procedure-related pain. J Burn Care Rehabil 1995;16:365-371

24. Dunbar PJ, Buckley P, Gavrin JR, et al. Use of patient-controlled analgesia for pain control for children receiving bone marrow transplant. J Pain Symptom Manage 1995;10:604-611.

25. Vanier MC, Labrecque G, Lepage-Savary D, et al. Comparison of hydromorphone continuous subcutaneous infusion and basal rate subcutaneous infusion plus PCA in cancer pain: a pilot study. Pain 1993;53:27-32.

26. Ferrell BR, Nash CC, Warfield C. The role of patient-controlled analgesia in the management of cancer pain. J Pain Symptom Manage 1992;7:149154.

27. Meuret G, Jocham H. Patient-controlled analgesia (PCA) in the domiciliary care of tumour patients. Cancer Treat Rev 1996;22:137-140.

28. Fainsinger RL, Nekolaichuk CL, Lawlor PG, et al. A multicenter study of the revised Edmonton Staging System for classifying cancer pain in advanced cancer patients. J Pain Symptom Manage 2005;29:224-237.

29. Mao JJ, Armstrong $\mathrm{K}$, Bowman MA, et al. Symptom burden among cancer survivors: impact of age and comorbidity. J Am Board Fam Med 2007;20:434-443.

30. Macintyre $P$, Jarvis D. Age is the best predictor of postoperative morphine requirements. Pain 1996;64:357-364

31. Fainsinger RL, Fairchild A, Nekolaichuk C, et al. Is pain intensity a predictor of the complexity of cancer pain management? J Clin Oncol 2009;27:585-590.

32. Novik A, Manikhas G, Ionova $\mathrm{T}$, et al. Comparison of symptom severity and prevalence between hematological malignancies and solid tumors among Russian oncology patients [abstract]. Blood 2002;100(Suppl 1):Abstract 387b

33. Niscola P, Arcuri E, Giovannini M, et al. Pain syndromes in haematological malignancies: an overview. Hematol J 2004;5:293-303.

34. Fadul NA, Osta BE, Dalal S, et al. Comparison of symptom burden among patients referred to palliative care with hematologic malignancies versus those with solid tumors. J Palliat Med 2008;11:422-427. 